\title{
Efecto de un Recubrimiento Comestible y de Diferentes Empaques durante el Almacenamiento Refrigerado de Mango Tommy Atkins Mínimamente Procesado
}

\author{
Saúl Dussán-Sarria*, Cristian Torres-León y Jose I. Hleap-Zapata \\ Fac. de Ingeniería, Depto. Ingeniería y Administración, Univ. Nacional de Colombia. Sede Palmira. A.A. \\ 237. Palmira, Valle del Cauca-Colombia. (e-mail: sdussan@unal.edu.co; ctorresl@unal.edu.co; \\ jihleapz@unal.edu.co)
}

${ }^{*}$ Autor a quien debe ser dirigida la correspondencia.

Recibido Oct. 31, 2013; Aceptado Dic. 30, 2013; Versión final recibida Feb. 27, 2014

\begin{abstract}
Resumen
Este trabajo tuvo como objetivo encontrar la condición más adecuada para el almacenamiento de mango Tommy Atkins mínimamente procesado. Se evaluó el efecto de un recubrimiento comestible de almidón de yuca y cera de carnauba adicionando ácidos orgánicos (1\%) y cloruro de calcio (1\%). Se estudió también el efecto de cuatro empaques. Se utilizó un diseño experimental completamente al azar con tres repeticiones, con un total de ocho tratamientos derivados de cuatro condiciones de empaques y dos de recubrimiento. Se aplicó estadística descriptiva, ANOVA y comparación de medias utilizando el ensayo Tukey. Se utilizó el software SAS $9.3(p<0,05)$. El fruto fue almacenado a $5 \pm 1^{\circ} \mathrm{C}$ y $90 \pm 2 \%$ de humedad relativa y se midió los atributos de calidad cada cuatro días, durante 24 días. El mango mínimamente procesado tratado con ácido cítrico, ácido ascórbico, cloruro de calcio y recubrimiento comestible, envasado en PET (tereftalato de polietileno) es la mejor condición para el almacenamiento de mango Tommy Atkins.
\end{abstract}

Palabras clave: alimento, frutas, Mangifera Indica, mínimamente procesado, empaque

\section{Effect of Edible Coating and Different Packaging during Cold Storage of Fresh-cut Tommy Atkins Mango}

\begin{abstract}
This objective of this work was to find the most suitable condition for the storage of fresh-cut Tommy Atkins mango. The effects of edible coating cassava starch and carnauba wax adding organic acids (1\%) and calcium chloride $(1 \%)$. were evaluated. Also the effect of four packaging materials was studied. The experimental design used was completely randomized with three replicates with a total of eight treatments derived from four packaging conditions and two of coatings. Descriptive statistics, ANOVA and means comparison using Tukey test were applied. The software SAS $9.3(p<0.05)$ was used. The fruit was stored at $5 \pm 1{ }^{\circ} \mathrm{C}$ and $90 \pm 2 \%$ of relative humidity and every four days for 24 days the quality attributes were measured. The fresh-cut mango treated with citric acid, ascorbic acid, calcium chloride and edible coating, using PET packaging (polyethylene terephthalate) is the most suitable condition for the storage of fresh-cut Tommy Atkins mango
\end{abstract}

Keywords: food, fruits, Mangifera Indica, fresh-cut, packaging 


\section{INTRODUCCIÓN}

El mango es una de las frutas tropicales más importantes en el mundo, principalmente por su agradable sabor, aroma y por su alto valor nutricional, es un fruto rico en agua, azúcares, fibra, minerales y vitaminas, (Singh et al., 2013). Los mangos 'Tommy Atkins' son frutos de gran tamaño (400-600 g), con cáscara roja brillante, sabor agradable y un gran potencial para la industria de procesamiento mínimo, ya que son resistentes al manejo agroindustrial y su pulpa es de color naranja-amarillo, con un alto contenido de vitaminas A, B y C (Chiumarelli et al., 2011). Existe una tendencia al consumo de alimentos inocuos y convenientes debido a los estilos modernos de vida y hábitos de consumo. La International Fresh-cut Produce Association (IFPA, 2004), define a los productos mínimamente procesados como "cualquier fruta o vegetal recién cortado.

La fruta cortada es muy perecedera debido a que pierde la protección del pericarpio, siendo modificadas sus propiedades fisicoquímicas por los trastornos metabólicos que se producen por el corte (Tovar et al, 2001), adicional a esto, este tipo procesamiento genera oscurecimiento y disminución de la firmeza (Chiumarelli et al., 2011). Por lo cual se hacen necesarios métodos alternativos para la preservación de los atributos de calidad del mango cortado para asegurar su manejo, distribución y venta. Muchas de las investigaciones se han centrado en el uso de tratamientos poscosecha para extender la vida útil, por ejemplo la adición de antioxidantes (ácido ascórbico), agentes reafirmantes (derivados de calcio), recubrimientos comestibles y envasado en atmósfera modificada con niveles reducidos de oxígeno (Kader, 2008). Estos agentes aplicados al mango minmamente procesadoubrimientos son comunmente utilizados para evaitar la decoloración del fruto, retener la firmeza, retener otros atributos ensoriales y prolongarl la vida útil del producto (Siddiq et al,.2012).Por su parte la aplicación de recubrimientos crea una barrera semipermeable a gases similar a una atmosfera modificada lo que reduce la velocidad de respiración y la deshidratación de los productos recubiertos (Chiumarelli et al., 2011). Todo esto con la ventaja de que el producto recubierto puede ser consumido en forma segura. También se han investigado diferentes tipos de tratamientos con recubrimientos comestibles y la aplicación o no de calor, con el fin de mantener las propiedades del mango fresco cortado por más tiempo (Sothornvit y Rodsamran, 2008; Djioua et al., 2009; Djioua et al., 2010; Souza et al., 2006; Chiumarelli et al., 2011). En este sentido y a través del método de solución tipo experimental, se tuvo como objetivo encontrar la condición agroindustrial más adecuada para el almacenamiento de mango mínimamente procesado y refrigerado.

\section{MATERIALES Y MÉTODOS}

El experimento se realizó en el laboratorio de frutas y hortalizas de la Universidad Nacional de Colombia (Palmira, Valle del Cauca, Colombia). Las experimentaciones se realizaron utilizando mangos variedad Tommy Atkins seleccionados y clasificados por tamaño uniforme y estado de madurez 2 según norma ICONTEC (2003). El mango destinado al procesamiento mínimo debe poseer color, firmeza interna y atributos de calidad para tal fin (Djioua et al., 2009; Chien et al., 2007).

Con ayuda de cuchillos en acero inoxidable desinfectados, se retiró la cáscara de los frutos y se realizaron cortes longitudinales, posterior a esto se fraccionaron las muestras para obtener finalmente trozos de fruta, el tipo de corte se obtuvo realizando pruebas preliminares hasta obtener el mayor rendimiento posible $(64,17 \%)$. Posteriormente todos los trozos de mango se sumergieron en hipoclorito de sodio a $10 \mathrm{ppm}$ durante 1 minuto, se dejaron escurrir por 2 min. Una vez realizada la higienización todos los trozos de mango utilizados en el experimento se sumergieron en una solución de ácido cítrico al 1\% + ácido ascórbico al $1 \%+1 \% \mathrm{CaCl}_{2}$ durante 3 minutos.

La formulación del recubrimiento comestible se basó en revisión de literatura y experimentación preliminar. La utilización de lípidos se basó en que las películas de almidón como recubrimientos de alimentos, tienen la dificultad de que son muy permeables al vapor de agua (Chiumarelli et al., 2011). Entonces, el uso de la cera de carnauba fue un complemento buscando reducir la higroscopicidad característica de los polisacáridos. Trabajos con formulaciones específicas que incluyan todos los compuestos comestibles utilizados en este trabajo, no se han desarrollado lo suficientemente en frutas perecederas cortadas como el mango.Para formar $1000 \mathrm{~g}$ de recubrimiento comestible se dispersaron $20 \mathrm{~g}$ almidón de yuca en $475 \mathrm{ml}$ de agua con agitación constante, esta mezcla se llevó a $75^{\circ} \mathrm{C}$ hasta su gelatinización. En otro recipiente, se adicionaron $15 \mathrm{~g}$ de glicerina en $475 \mathrm{ml}$ de agua destilada, esta mezcla se le incorporó gota a gota al gel de almidón ya formado, esta solución final se mantuvo en agitación constante durante 10 minutos. Posterior a esto se adicionó gota a gota una solución de ácido esteárico ( $8 \mathrm{~g})$, cera de carnauba $(2 \mathrm{~g})$ y aceite de canola $(4 \mathrm{~g})$ diluidos a $85^{\circ} \mathrm{C}$, con el fin de formar la emulsión. Finalmente se dejó el sistema en agitación constante por 3 minutos a $85^{\circ} \mathrm{C}$, luego se llevó a temperatura ambiente. Las muestras de mango cortado una vez aplicado los antioxidantes y el calcio, se dividieron en dos grupos, uno se dejó sin recubrimiento y el otro se sumergió durante 2 minutos en el recubrimiento comestible formado. 
Una vez aplicado el recubrimiento comestible ambas muestras de mango en cantidad de $150 \mathrm{~g}$ aproximadamente, se acondicionaron en cuatro (4) envases diferentes: bolsa de polietileno de alta densidad $(19 \mathrm{~cm} \times 14 \mathrm{~cm})$ calibre $70 \mu \mathrm{m}$ aplicando vacío, bolsa de polietileno de baja densidad $(15 \mathrm{~cm} \times 15 \mathrm{~cm})$ calibre $40 \mu \mathrm{m}$, bandeja de Icopor $(10 \mathrm{~cm} \times 10 \mathrm{~cm}$ ) envuelta con policloruro de vinilo (PVC) y cajas PET (Tereftalato de polietileno) de medidas $(12 \mathrm{~cm} \times 8 \mathrm{~cm} \times 4 \mathrm{~cm})$. El fruto mínimamente procesado y acondicionado se almacenó a Temperatura de $5 \pm 1^{\circ} \mathrm{C}$ y $85 \%$ a $90 \%$ de HR (Sothornvit y Rodsamran, 2008; Kader, 2008).

\section{EVALUACIÓN DE ATRIBUTOS}

A las muestras de mango mínimamente procesadas se le realizaron análisis los días $0,4,8,12,16,20,24$ de almacenamiento.

\section{Análisis sensorial}

A través de 30 jurados no entrenados se evaluaron sensorialmente cambios de apariencia externa y sabor del mango en trozos. Se consideró un análisis sensorial afectivo de apariencia y aceptabilidad de acuerdo en la siguiente escala de notas: 5 me gusta mucho, 4 me gusta, 3 ni me gusta ni me disgusta, 2 me gusta poco, 1 no me gusta. Los cortes se consideraron sensorialmente aceptables con nota mínimo de 3 (Souza et al., 2006).

\section{Parámetros de color}

El color de la superficie de los trozos de mango se midió utilizando un colorímetro Marca Konica Minolta, Modelo: CR - 400, escala $\left(L^{*}, a^{*}, b^{*}\right)$, D65 como fuente luminosa y $10^{\circ}$ ángulo de observación, Las mediciones de color se expresan en términos de luminosidad $L^{*}\left(L^{*}=0\right.$ para el negro y $L^{*}=100$ para el blanco), y los parámetros de cromaticidad $a^{*}$ (verde [-], rojo [+]) y $b^{*}$ (azul[-], amarillo [+]). A partir de estos parámetros, fueron calculadas las coordenadas $\mathrm{C}^{*}$ (croma) y $\mathrm{H}^{*}$ (tono) utilizando la ecuación 1 y la ecuación 2, respectivamente. Las mediciones se realizaron por triplicado (Chiumarelli et al., 2011)

$$
\begin{aligned}
& C=\left(a^{2}+b^{2}\right)^{1 / 2} \\
& H=\operatorname{artan}\left(\frac{b}{a}\right)
\end{aligned}
$$

\section{Firmeza}

La firmeza se midió manualmente con un penetrometro (FORCE DIAL FDK 30, capacidad: 14kgf x 100gf (Italia). Se determinó midiendo la fuerza requerida para penetrar una punta de $8 \mathrm{~mm}$ de diámetro en dirección perpendicular a la superficie de la muestra de mango, los valores se expresan Kgf) (Djioua et al., 2009).

\section{RESULTADOS Y DISCUSIÓN}

Se evaluó 8 tratamientos derivados de cuatro (4) condiciones de envase y dos (2) de recubrimiento (con recubrimiento comestible y sin recubrimiento comestible), designados de la siguiente forma:

Sin Recubrimiento Comestible: PVC, VACIO, BOLSA, PET

Con Recubrimiento Comestible: PVC-R; VACIO-R; BOLSA-R; PET-R

La unidad experimental fue cada envase conteniendo el fruto cortado. Se utilizó el delineamiento experimental completamente al azar con tres repeticiones de cada tratamiento y se aplicó análisis estadístico basado en estadística descriptiva, análisis de varianza y comparación de medias utilizando el ensayo Tukey. Estos valores se analizaron a través del software SAS 9.3 con un nivel de significancia $p<$ 0,05 .

\section{Análisis sensorial}

La calidad organoléptica del mango mínimamente procesado (MP) disminuye notablemente después del octavo día de almacenamiento en todos los tratamientos, siendo mucho más acentuada en mango cortado sin recubrimiento comestible (Figura 1). La mayor pérdida de apariencia y de aceptabilidad sensorial se presentó en la bandeja de poliestireno recubierta con PVC con y sin recubrimiento comestible. La aplicación 
previa de los antioxidantes ácido cítrico y ácido ascórbico, evitaron el pardeamiento enzimático prematuro en todos los tratamientos, siendo más eficaz en cuanto a la apariencia y aceptabilidad del mango en trozos (notas de 3 a 5) acondicionado en PET con recubriendo (PET-R), conservando la calidad sensorial hasta el día 24 de almacenamiento refrigerado (Figura 1). La apariencia es un criterio muy importante para determinar la aceptabilidad de los productos. Esta es utilizada como un indicador de frescura y calidad en los productos mínimamente procesados tanto en la investigación científica como en la industria (Djioua et al., 2009).

Previamente Dang et al. (2008), reportaron que mangos de la variedad Kensington pride recubiertos con ceras de carnauba presentaron una mejora en la composición de compuestos volátiles. Es importante destacar que otro factor que ayudo a que la utilización del recubrimiento no afectara la apariencia de las muestras es el hecho que los recubrimientos a base de almidón son insípidos, inodoros y transparentes, por la tanto no cambian el sabor, aroma o apariencia del producto (Chiumarelli y Hubinger, 2012). La apariencia es un criterio muy importante para determinar la aceptabilidad de los productos. Es utilizada como un indicador de frescura y calidad en los productos mínimamente procesados tanto en la investigación científica como en la industria (Djioua et al., 2009).

Con respecto a los empaques, la utilización de PVC reporta los valores más bajos en la escala sensorial de aceptación seguido por el mango acondicionado en bolsas de polietileno de $40 \mu \mathrm{m}$. En el empaque al vacío se observó un efecto sensorial negativo en la apariencia provocado por el recubrimiento sobre el mango cortado (imágenes no presentadas).
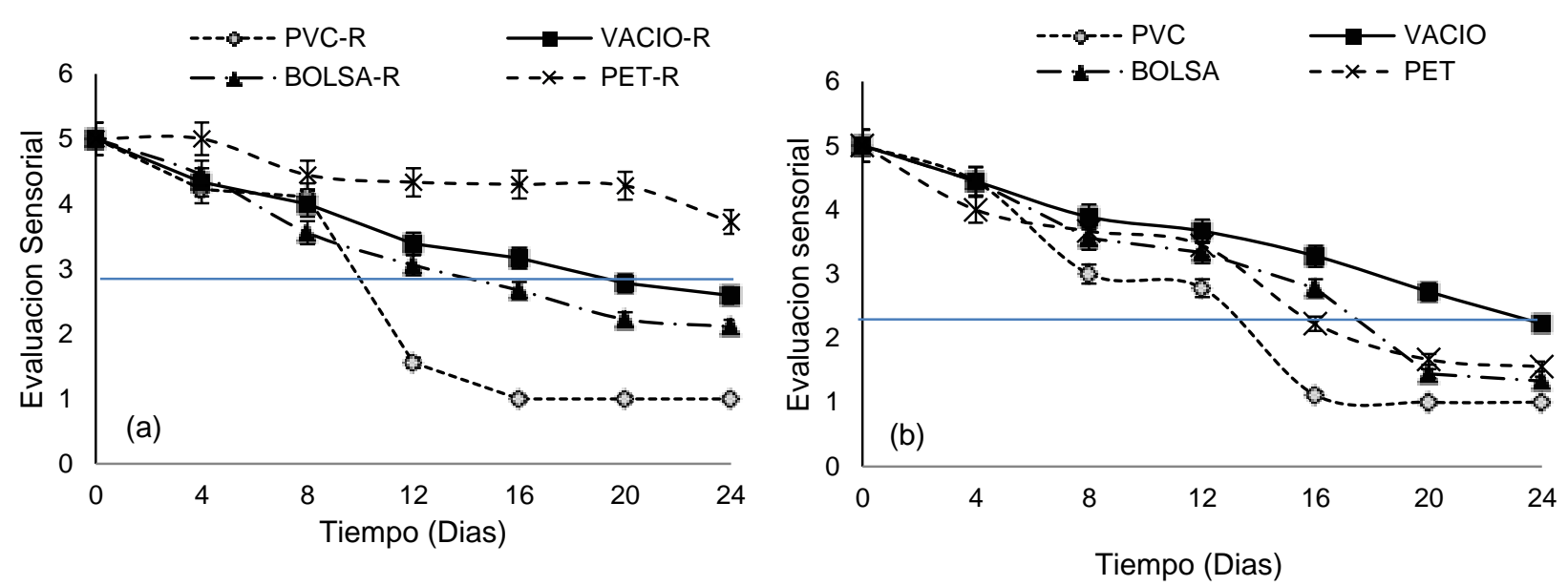

Fig. 1. Análisis sensorial de mango MP. Con recubrimiento comestible (a) y sin recubrimiento comestible (b), acondicionado en diferentes empaques y almacenado a $5 \pm 1^{\circ} \mathrm{C}$ y $90 \pm 2 \%$ de HR.

\section{Color}

Las mediciones de color se expresan en términos de luminosidad $L^{*}\left(L^{*}=0\right.$ para el negro y $L^{*}=100$ para el blanco), y los parámetros de cromaticidad $a^{*}$ (verde [-], rojo [+]) y $b^{*}$ (azul [-], amarillo [+]). A partir de estos parámetros, fueron calculadas las coordenadas $\mathrm{C}^{*}$ (croma) y $\mathrm{H}^{*}$ (tono).

Las Figuras 2, 3 y 4 presentan los cambios en la luminosidad $\left(L^{*}\right)$, cromaticidad: saturación $\left(C^{*}\right)$ y tonalidad $\left(\mathrm{H}^{*}\right)$ del mango MP durante el almacenamiento. En general los tratamientos con recubrimiento comestible no presentan diferencias significativas en los resultados de color $(p<0,05)$, esto indica que la utilización del recubrimiento no afecto significativamente los cambios de color del mango, contrario con lo que se encontró con el mango cortado sin recubrimiento comestible. Robles et al. (2013), encontraron en cubos de mango de la variedad Kent buenos resultados en los parámetros de color, utilizando recubrimiento comestible con antioxidantes, al final del almacenamiento las muestras con recubrimiento solo perdieron $2,5 \%$ de su valor inicial de $L^{*}$ en comparación con una pérdida del $7 \%$ en las muestras que no tenían recubrimiento comestible.

El tratamiento que evidenció mayor pérdida de luminosidad $\left(L^{*}\right)$ fue el de mango acondicionado en bandeja de poliestireno con PVC sin recubrimiento comestible. Los tratamientos de mango cortado con recubrimiento y sin recubrimiento, almacenado en el empaque PET presentaron los mayores valores de luminosidad durante el almacenamiento. 

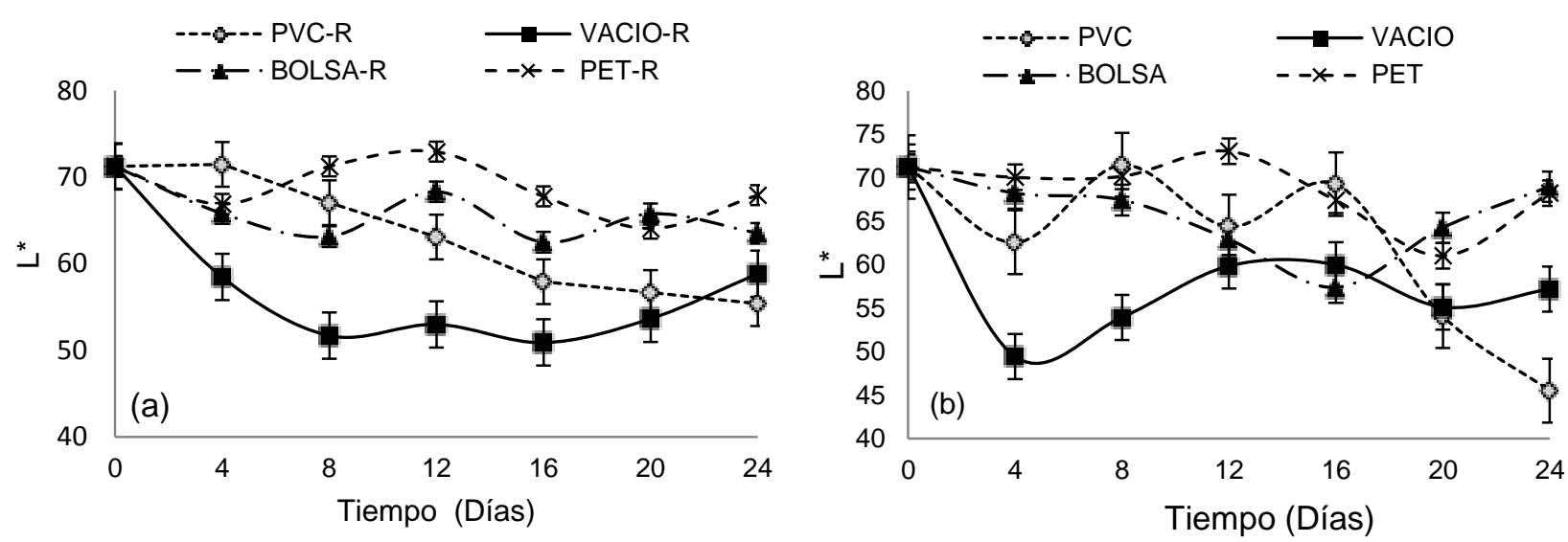

Fig. 2. Cambios en la luminosidad $\left(L^{*}\right)$ de mango MP. Con recubrimiento comestible (a) y sin recubrimiento comestible (b), acondicionado en diferentes empaques y almacenado a $5 \pm 1^{\circ} \mathrm{C}$ y $90 \pm 2 \%$ de $\mathrm{HR}$.

Dado que los recubrimientos comestibles a base de polisacáridos como el almidón son una buena barrera a los gases, con su utilización se logra disminuir la tasa de respiración y las actividades metabólicas, logrando así un retraso efectivo en el pardeamiento. El recubrimiento de almidón de yuca, ácido cítrico y glicerol ofrecen un efectivo mantenimiento de las características de color en muestras de mango cortado, debido al efecto combinado del recubrimiento y el ácido cítrico (Chiumarelli et al., 2011). El ácido ascórbico y el cítrico tienen la capacidad de inhibir la acción de PPO que funciona como agente de reducción (o-quinonas a difenoles) y como reductor de $\mathrm{pH}$ (Hu et al., 2007), mejorando el efecto de los recubrimientos en la prevención del pardeamiento de las muestras de mango.

Los valores de Croma $\left(\mathrm{C}^{*}\right)$ y Tono $\left(\mathrm{H}^{*}\right)$, son considerados valores importantes en este trabajo debido a que incluyen las variaciones en las longitudes de onda $a^{*}$ y $b^{*}$ abarcando el espectro de color del mango en su proceso de maduración (color crema hasta amarillo intenso). Se observó que los valores de $\mathrm{C}^{\star}$ se mantienen constantes con ciertas variaciones en el tiempo, no habiendo diferencia estadísticas entre los tratamientos con y sin recubrimiento comestible (Figura 3a y Figura 3b). Se destaca que el mango MP acondicionado en la bolsa de polietileno seguido por el PET con y sin recubriendo comestible presentaron en media los mayores valores de Croma durante todo el periodo de almacenamiento.
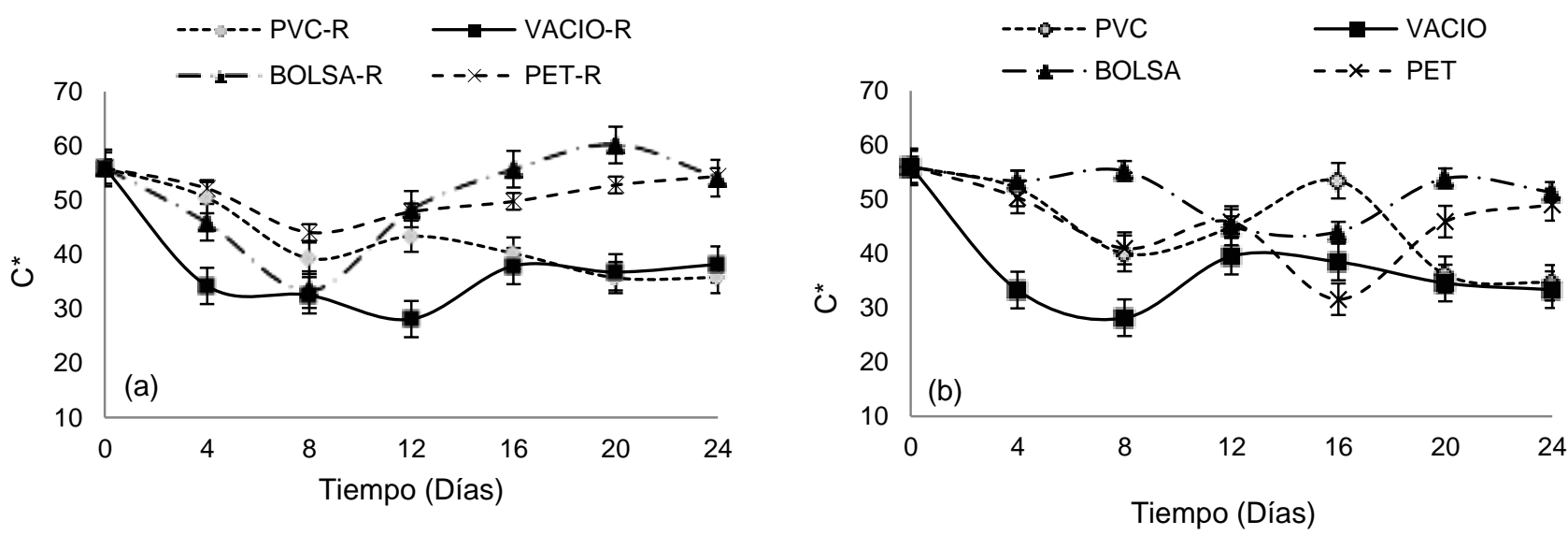

Fig. 3. Cambios en la cromaticidad (croma ${ }^{*} \mathrm{C}$ ) de mango MP. Con recubrimiento comestible (a) y $\sin$ recubrimiento comestible (b), acondicionado en diferentes empaques y almacenado a $5 \pm 1^{\circ} \mathrm{C}$ y $90 \pm 2 \%$ de HR

Los valores de Tono $\left(\mathrm{H}^{*}\right)$ fueron muy cercanos y constantes en todos los tratamientos durante el almacenamiento refrigerado, siendo el tratamiento de mango MP en bandeja de poliestireno mas PVC el que menores valores exhibió durante la conservación (Figura 4a y Figura 4b). 

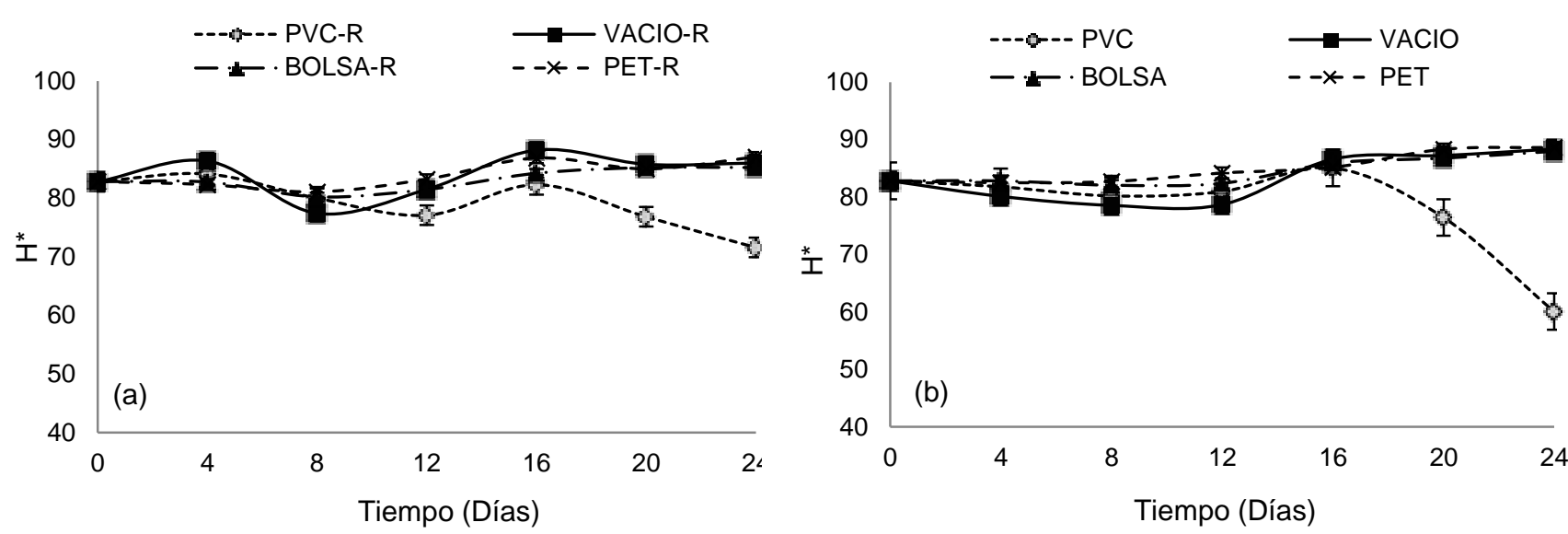

Fig. 4. Cambios en la cromaticidad (tono $\mathrm{H}^{*}$ ) de mango MP. Con recubrimiento comestible (a) y sin recubrimiento comestible (b), acondicionado en diferentes empaques y almacenado a $5 \pm 1^{\circ} \mathrm{C}$ y $90 \pm 2 \%$ de HR.

\section{Firmeza}

Debido a la aplicación previa de cloruro de calcio en todos los tratamientos con y sin recubrimiento comestible, se considera que el calcio fue incorporado a los tejidos del producto y tuvo un efecto importante y positivo sobre los valores de la firmeza en el mango MP acondicionado al vacío y en PET, en los primeros 4 días de almacenamiento, con un valor inicial en el día cero de 1,5 $\mathrm{kgf}$ y en el día 4 de aproximadamente 3 kgf. En los demás tratamientos no se observó este comportamiento. Después del día 4 y hasta el día 24 de almacenamiento el tratamiento que mejor conservó la firmeza del mango MP fue el acondicionado en PET con recubrimiento comestible, encontrándose un valor de firmeza al finalizar el almacenamiento de 2,7kgf (Figura 5). El tratamiento con ácido cítrico, ácido ascórbico y la adición de calcio, ya había presentado buenos resultados al mantener la firmeza del mango cortado (Siddiq et al., 2013).

De acuerdo con lo reportado por Souza et al. (2006), el tratamiento con calcio aumenta la firmeza inicial de cubos de mango en los 3 primeros días de almacenamiento comportamiento que va disminuyendo. Rojas Grau et al. (2008), encontraron en recubrimientos comestibles un efecto similar en manzanas, reportando que este ayuda a evitar el deterioro de la firmeza.
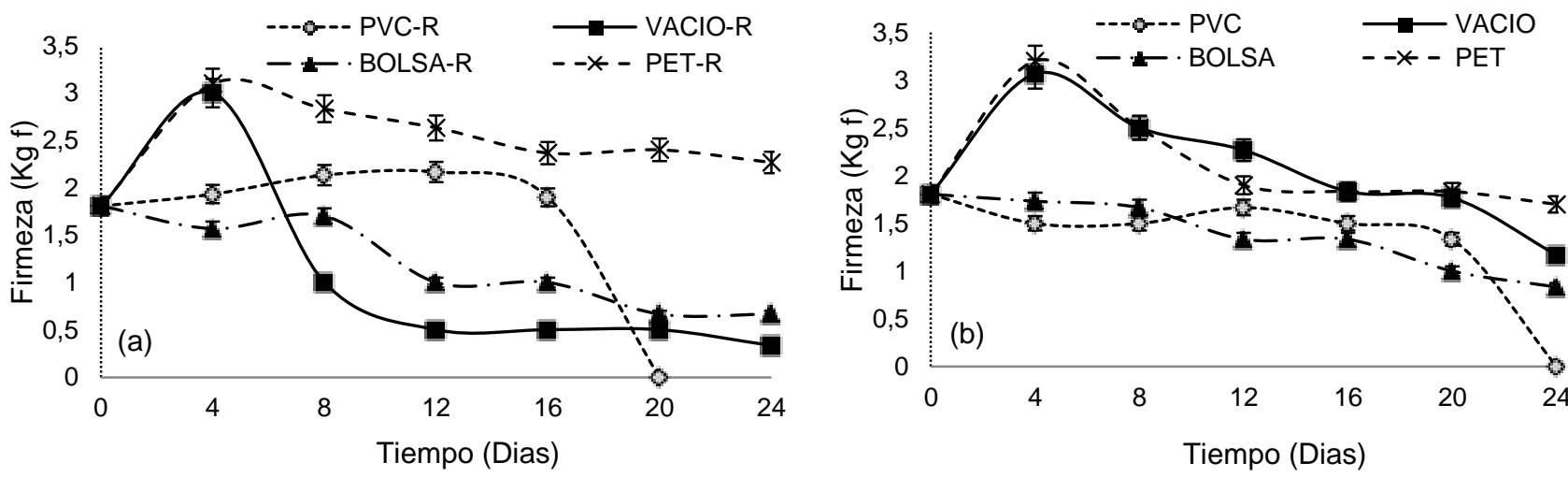

Fig. 5. Cambios en los valores de firmeza de mango MP. Con recubrimiento comestible (a) y sin recubrimiento comestible (b), acondicionado en diferentes empaques y almacenado a $5 \pm 1^{\circ} \mathrm{C}$ y $90 \pm 2 \%$ de HR.

Por otra parte la heterogeneidad en los frutos y la falta de uniformidad en la estructura interna del material biológico provocada por la dificultad de homogenizar inicialmente en la experimentación frutos con idéntico grado de maduración, promueve desviaciones en los valores de firmeza en diferentes materias primas de origen biológico (Chiumarelli et al., 2011; Chiumarelli y Hubinger, 2012), esto puede explicar en parte la des uniformidad en los valores graficados en la Figura 5. 
De acuerdo al análisis estadístico las diferencias encontradas en la apariencia, color y firmeza son debidas a los empaques y no al tener o no recubrimiento comestible. El empaque PET contribuyó de forma positiva a la conservación de todas las propiedades de color, apariencia, firmeza y aceptabilidad sensorial en el mango MP, esto seguramente debido a la efectiva atmósfera modificada originada por el envase y la aplicación del recubrimiento, es decir, en una atmósfera baja en oxígeno y con elevada concentración de $\mathrm{CO}_{2}$, evitando que suceda oxidación o degradación de carotenoides (pigmentos vegetales inestables en la presencia de oxigeno) durante el almacenamiento.

Con base a todos los atributos de calidad analizados anteriormente, se unificaron los valores de cada uno de los atributos en la Tabla 1. Estimando la vida útil en días para cada tratamiento.

Tabla 1. Estimación de la vida útil de mango mínimamente procesado tratado o no con recubrimiento comestible.

\begin{tabular}{|l|c|r|r|r|r|r|}
\hline \multirow{2}{*}{ Tratamiento } & \multirow{2}{*}{ Vida útil (Días) } & \multirow{2}{*}{ Sensorial } & \multicolumn{3}{|c|}{ Color } & \multirow{2}{*}{ Firmeza (kgf) } \\
\cline { 4 - 6 } & & & ${ }^{*} \mathrm{~L}$ & ${ }^{*} \mathrm{C}$ & ${ }^{*} \mathrm{H}$ & \\
\hline PVC & 8 & 3 & 71,5 & 40,0 & 80,2 & 1,5 \\
\hline PVC-R & 10 & 3 & 65,1 & 41.4 & 78,5 & 2,1 \\
\hline VACIO & 18 & 3 & 61,7 & 36,5 & 86,9 & 1,8 \\
\hline VACIO-R & 18 & 3 & 52,3 & 37.3 & 86,9 & 0,5 \\
\hline PET & 14 & 3 & 70,3 & 38,7 & 84,6 & 1,9 \\
\hline PET-R & 24 & 3,7 & 70,3 & 54.4 & 87,0 & 2,3 \\
\hline BOLSA & 13 & 3 & 64,0 & 44,0 & 83,0 & 1,3 \\
\hline BOLSA-R & 13 & 3 & 69,2 & 49,0 & 82,5 & 1,0 \\
\hline
\end{tabular}

Al final de la experimentación en el mango acondicionado en PET-R resultó ser un producto con características sensoriales agradables en cuanto a sabor y aroma. El efecto positivo del empaque PET en el mango MP, sumado a la aplicación de recubrimiento comestible, conserva las propiedades de color, apariencia, aceptabilidad sensorial y desaceleración del proceso metabólico de la maduración que incluye los atributos de calidad evaluados, esto es debido a la efectiva atmósfera modificada lograda por el cierre hermético del envase y la aplicación del recubrimiento. Este efecto positivo en la prolongación de la vida anaquel de trozos de mango almacenados en empaques de polieteilenoterftalato (PET) ha sido reportado por (Donadon y Durigan 2004), (Singh et al., 2007) y (Chonhenchob et al., 2007), los cuales concluyen que la vida en anaquel de mangos pre cortados puede ser extendida en empaques de PET, debido a la reducción de oxígeno y aumento en la concentración de dióxido de carbono. Este estudio evidencia que se pueden obtener productos de fácil consumo y con un largo tiempo de almacenamiento, lo que proyecta esta tecnología como muy prometedora en el mango cortado, pude utilizarse tanto en países desarrollados como en vía de desarrollo (Singh et al., 2013).

\section{CONCLUSIONES}

La técnica agroindustrial integral más adecuada de mango mínimamente procesado y que permite prolongar su vida útil hasta por 24 días a condiciones de refrigeración $\left(5 \pm 1^{\circ} \mathrm{C}\right.$ y $90 \pm 2 \%$ de HR), consiste en realizar inmersión en ácido ascórbico $(1 \% \mathrm{v} / \mathrm{v})$, ácido cítrico $(1 \% \mathrm{v} / \mathrm{v})$ y $\mathrm{CaCl}_{2}(1 \% \mathrm{v} / \mathrm{v})$, posteriormente realizar el recubrimiento comestible a base de almidón de yuca, glicerol, cera de carnauba y aceite de canola y finalmente el acondicionamiento en empaque PET (polietileno tereftalato). La utilización de un recubrimiento comestible complementado con un envase adecuado como lo fue el PET, contribuye a mantener los atributos de calidad sensorial, físicos y químicos del mango MP bajo refrigeración.

\section{REFERENCIAS}

Chien, P., Sheu F. y Yang F. Effects of Edible Chitosan Coating on Quality and Shelf Life of Sliced Mango Fruit. Journal of Food Engineering, 78 (1) 225-229 (2007).

Chiumarelli, M., Cristhiane C. F., Sarantópoulos C. y Hubinger M. Fresh Cut 'Tommy Atkins' Mango Pretreated with Citric Acid and Coated with Cassava (Manihot Esculenta Crantz) Starch or Sodium Alginate. Innovative Food Science \& Emerging Technologies 12 (3) 381-387. (2011).

Chiumarelli, M. y Hubinger M., Stability, Solubility, Mechanical and Barrier Properties of Cassava Starch Carnauba Wax Edible Coatings to Preserve Fresh-cut Apples. Food Hydrocolloids 28 (1), 59-67 ( 2012). 
Chonhenchob, V., Chantarasomboon, Y. y Singh S. Quality Changes of Treated Fresh- Cut Tropical Fruits in Rigid Modified Atmosphere Packaging Containers. Technol. Sci. 20, 27-37 (2007).

Djioua, T., Charles, F., Lopez-Lauri, F., Filgueiras, H., Coudret A., Freire M., Ducamp-Collin, M. y Sallanon H. Improving the Storage of Minimally Processed Mangoes (Mangifera Indica L.) by Hot Water Treatments. Postharvest Biology and Technology 52 (2) 221-226 (2009).

Djioua, T., Charles F., Freire M, Filgueiras H., Ducamp-Collin, M. y Sallanon H. Combined Effects of Postharvest Heat Treatment and Chitosan Coating on Quality of Fresh-cut Mangoes (Mangifera Indica L.). International Journal of Food Science \& Technology, 45 (4) 849-855 (2010).

Donadon, J.R., y Durigan J. F. Production and Preservation of Fresh-cut 'Tommy Atkins' Mango Chunks." Acta Hort, 645, 257-260 (2004).

Hu, W., Jiang, A., Qi, H., Pang, K. y Fan S. Effects of initial low oxygen and perforated film package on quality of fresh-cut cabbages. Journal of the Science of Food and Agricultural, 87, 2019-2025 (2007).

ICONTEC (2003): Norma Técnica Colombiana 5210. Frustas frescas. Mango variedades mejoradas. Especificaciones.

International Fresh-cut Produce Association. 'Fresh-cut Produce Fuels An America On-the-go', IFPA, 2004. http://www.fresh-cuts.org. Acceso: 12 de febrero (2014).

Kader, A. A., Fresh-cut Mangos as a Value-added Product (Literature Review). 2008. http://www.mango.org/es/industry/research/producto-fresco-cortado-como-producto-de-valor-agregadoconsulta-literaria. Orlando, FL, USA. Acceso: 20 de marzo (2013).

Oliveira, E. S., Durigan, J. F. y Alves S. A. P. 2003. "Processamento Mínimo de Frutas." 2003. http://www.cnpat.embrapa.br. Acceso: 12 de febrero (2014).

Robles, R., Rojas, M., Odriozola, I., Gonzales., G, Martin, O. Influence of alginate-based edible coating as carrier of antibrowning agents on bioactive compounds and antioxidant activity in fresh-cut Kent mangoes. Food Science and Technology.50, 240-246 (2013).

Rojas - Grau, M., Tapia M., y Belloso O. Using Polysaccharide-based Edible Coatings to Maintain Quality of Fresh-cut Fuji Apples. LWT - Food Science and Technology, 41, 139-147 (2008).

Siddiq M., Akhtar, S. y Siddiq R. Mango Pocessing, Products and Nutrition. In: Siddiq, M. Tropical and Subtripical Fruits. Posthrvest and Physiology, Processing and Packaging. 1a edición. 277-284. lowa, USA. 2012.

Siddiq, M., Sogi, D., K. Dolan. Antioxidant properties, total phenolics, and quality of fresh-cut 'Tommy Atkins' mangoes as affected by different pre-treatments. Food Science and Technology, 53, 156-162 (2013).

Singh, S., Chonhenchob, V. y Singh, J. Testing and Evaluation of Quality Changes of Treated Fresh-cut Tropical Fruits Packaged in Thermoformed Plastic Containers. Journal of Testing and Evaluation, 35 (5) 522-528 (2007).

Singh, Z., Singh, R., Sane, V. y Nath, P. Mango - Postharvest Biology and Biotechnology. Critical Reviews in Plant Sciences, 32(4), 217-236 (2013)

Sothornvit, R. y Rodsamran P. Effect of a Mango Film on Quality of Whole and Minimally Processed Mangoes. Postharvest Biology and Technology, 47 (3) 407-415 (2008).

Souza, B. S., O'Hare, T. J., Durigan, J. F. y Souza, P. S. Impact of Atmosphere, Organic Acids, and Calcium on Quality of Fresh-cut 'Kensington' Mango. Postharvest Biology and Technology, 42 (2) 161-167 (2006):

Tovar, B., Garcia, H. S. y Mata, M. Physiology of Pre-cut Mango II . Evolution of Organic Acids. Food Research International 34, 705-714 (2001). 